\title{
Arguments in Favor of the Riemann Hypothesis
}

\author{
Frank Vega
}

CopSonic, 1471 Route de Saint-Nauphary 82000 Montauban, France

\begin{abstract}
The Riemann hypothesis is a conjecture that the Riemann zeta function has its zeros only at the negative even integers and complex numbers with real part $\frac{1}{2}$. The Riemann hypothesis belongs to the David Hilbert's list of 23 unsolved problems. Besides, it is one of the Clay Mathematics Institute's Millennium Prize Problems. This problem has remained unsolved for many years. The Robin criterion states that the Riemann hypothesis is true if and only if the inequality $\sigma(n)<e^{\gamma} \times n \times \log \log n$ holds for all natural numbers $n>5040$, where $\sigma(x)$ is the sumof-divisors function and $\gamma \approx 0.57721$ is the Euler-Mascheroni constant. The Nicolas criterion states that the Riemann hypothesis is true if and only if the inequality $\prod_{q \leq q_{n}} \frac{q}{q-1}>e^{\gamma} \times \log \theta\left(q_{n}\right)$ is satisfied for all primes $q_{n}>2$, where $\theta(x)$ is the Chebyshev function. Using both inequalities, we show some arguments in favor of the Riemann hypothesis is true.
\end{abstract}

Keywords: Riemann hypothesis, Robin inequality, Nicolas inequality, sum-of-divisors function, Chebyshev function, prime numbers, Riemann zeta function

2000 MSC: 11M26, 11A41, 11A25

\section{Introduction}

In mathematics, the Chebyshev function $\theta(x)$ is given by

$$
\theta(x)=\sum_{q \leq x} \log q
$$

where $q \leq x$ means all the prime numbers $q$ that are less than or equal to $x$. Let $N_{n}=2 \times 3 \times$ $5 \times 7 \times 11 \times \cdots \times q_{n}$ denotes a primorial number of order $n$ such that $q_{n}$ is the $n^{\text {th }}$ prime number. Thus, $\theta\left(q_{n}\right)=\log N_{n}$. We define a sequence based on this function:

Definition 1.1. For every prime number $q_{n}$, we define the sequence of real numbers:

$$
X_{n}=\frac{\prod_{q \leq q_{n}} \frac{q+1}{q}}{\log \theta\left(q_{n}\right)} .
$$

We use this limit superior,

Email address: vega.frank@gmail.com (Frank Vega) 
Theorem 1.2. [1].

$$
\limsup _{n \rightarrow \infty} X_{n}=\frac{e^{\gamma} \times 6}{\pi^{2}}
$$

Say Nicolas $\left(q_{n}\right)$ holds provided

$$
\prod_{q \leq q_{n}} \frac{q}{q-1}>e^{\gamma} \times \log \theta\left(q_{n}\right) .
$$

The constant $\gamma \approx 0.57721$ is the Euler-Mascheroni constant and log is the natural logarithm. The importance of this inequality is:

Theorem 1.3. Nicolas $\left(q_{n}\right)$ holds for all prime numbers $q_{n}>2$ if and only if the Riemann hypothesis is true [2].

As usual $\sigma(n)$ is the sum-of-divisors function of $n[1]$ :

$$
\sum_{d \mid n} d
$$

where $d \mid n$ means the integer $d$ divides $n$ and $d \nmid n$ signifies that the integer $d$ does not divide $n$. Define $f(n)$ to be $\frac{\sigma(n)}{n}$. Say Robins $(n)$ holds provided

$$
f(n)<e^{\gamma} \times \log \log n .
$$

The importance of this inequality is:

Theorem 1.4. Robins(n) holds for all natural numbers $n>5040$ if and only if the Riemann hypothesis is true [3]. If the Riemann hypothesis is false, then there are infinitely many natural numbers $n>5040$ such that $\operatorname{Robins}(n)$ does not hold [3].

It is known that Robins $(n)$ holds for many classes of numbers $n$. We recall that an integer $n$ is said to be square free if for every prime divisor $q$ of $n$ we have $q^{2} \nmid n$ [1].

Theorem 1.5. Robins $(n)$ holds for all natural numbers $n>5040$ that are square free [1].

Let $q_{1}=2, q_{2}=3, \ldots, q_{m}$ be the first $m$ consecutive primes, then an integer of the form $\prod_{i=1}^{m} q_{i}^{a_{i}}$ with $a_{1} \geq a_{2} \geq \cdots \geq a_{m} \geq 0$ is called an Hardy-Ramanujan integer [1]. Based on the theorem 1.4, we know this result:

Theorem 1.6. If the Riemann hypothesis is false, then there exist infinitely many natural numbers $n>5040$ which are an Hardy-Ramanujan integer and Robins $(n)$ does not hold [1].

We define $H=\gamma-B$ such that $B \approx 0.2614972128$ is the Meissel-Mertens constant [4]. For all real numbers $x \geq 2$, the function $u(x)$ is defined as follows

$$
u(x)=\sum_{q>x}\left(\log \left(\frac{q}{q-1}\right)-\frac{1}{q}\right) .
$$

For all real numbers $x>1$, we define:

$$
\delta(x)=\left(\sum_{q \leq x} \frac{1}{q}-\log \log x-B\right) .
$$


Definition 1.7. We define another function:

$$
\varpi(x)=\left(\sum_{q \leq x} \frac{1}{q}-\log \log \theta(x)-B\right)
$$

for all real numbers $x \geq 3$.

Putting all together we provide some arguments in favor of the Riemann hypothesis is true.

\section{Known Results}

We know from the constant $H$, the following formula:

Theorem 2.1. [1].

$$
\sum_{q}\left(\log \left(\frac{q}{q-1}\right)-\frac{1}{q}\right)=\gamma-B=H .
$$

We know this property for the Chebyshev function:

Theorem 2.2. [5].

$$
\lim _{x \rightarrow \infty} \frac{\theta(x)}{x}=1
$$

Mertens second theorem states that:

Theorem 2.3. [4].

$$
\lim _{x \rightarrow \infty} \delta(x)=0 .
$$

We know these properties for the function $f(n)$ :

Theorem 2.4. [6]. Let $\prod_{i=1}^{m} q_{i}^{a_{i}}$ be the representation of $n$ as a product of primes $q_{1}<\cdots<q_{m}$ with natural numbers as exponents $a_{1}, \ldots, a_{m}$. Then,

$$
f(n)=\left(\prod_{i=1}^{m} \frac{q_{i}}{q_{i}-1}\right) \times \prod_{i=1}^{m}\left(1-\frac{1}{q_{i}^{a_{i}+1}}\right) .
$$

Theorem 2.5. [1]. For all natural numbers $n>1$ :

$$
f(n)<\prod_{q \mid n} \frac{q}{q-1} .
$$

We know these results for the Riemann zeta function:

Theorem 2.6. [7].

$$
\prod_{k=1}^{\infty} \frac{1}{1-\frac{1}{q_{k}^{2}}}=\prod_{k=1}^{\infty} \frac{q_{k}^{2}}{q_{k}^{2}-1}=\zeta(2)=\frac{\pi^{2}}{6} .
$$

Theorem 2.7. [7]. For $a \geq 1$ :

$$
\prod_{q}\left(1-\frac{1}{q^{a+1}}\right)=\frac{1}{\zeta(a+1)} .
$$

Finally, we know that:

Theorem 2.8. [2]. For all real numbers $x \geq 2$ :

$$
0<u(x) \leq \frac{1}{2 \times(x-1)}
$$




\section{Ancillary lemmas}

The following is a key lemma. It gives an upper bound on $f(n)$ that holds for all natural numbers $n$. The bound is too weak to prove Robins $(n)$ directly, but is critical because it holds for all natural numbers $n$. Further the bound only uses the primes that divide $n$ and not how many times they divide $n$.

Lemma 3.1. Let $n>1$ and let all its prime divisors be $q_{1}<\cdots<q_{m}$. Then,

$$
f(n)<\frac{\pi^{2}}{6} \times \prod_{i=1}^{m} \frac{q_{i}+1}{q_{i}} .
$$

Proof. Putting together the theorems 2.5 and 2.6 yields the proof:

$$
f(n)<\prod_{i=1}^{m}\left(\frac{q_{i}}{q_{i}-1}\right)=\prod_{i=1}^{m}\left(\frac{q_{i}+1}{q_{i}} \times \frac{1}{1-\frac{1}{q_{i}^{2}}}\right)<\frac{\pi^{2}}{6} \times \prod_{i=1}^{m} \frac{q_{i}+1}{q_{i}} .
$$

The following is another key lemma.

Lemma 3.2. There exists a natural number $N$ such that $X_{n}<\frac{e^{\gamma} \times 6}{\pi^{2}}+\varepsilon$ for all natural numbers $n>N$ and for a positive real number $\varepsilon<\frac{6}{\pi^{2}}$. Only a finite number of elements of the sequence are greater than $\frac{e^{\gamma} \times 6}{\pi^{2}}+\varepsilon$ (this could be an empty set).

Proof. The limit superior of a sequence of real numbers $y_{n}$ is the smallest real number $b$ such that, for any positive real number $\varepsilon$, there exists a natural number $N$ such that $y_{n}<b+\varepsilon$ for all natural numbers $n>N$. Only a finite number of elements of the sequence are greater than $b+\varepsilon$ (this could be an empty set). Therefore, this is a consequence of the theorem 1.2.

This is also a helpful lemma.

\section{Lemma 3.3.}

$$
\sum_{q}\left(\frac{1}{q}-\log \left(1+\frac{1}{q}\right)\right)=\log \left(\frac{\pi^{2}}{6}\right)-H .
$$

Proof. If we add $H$ to

$$
\sum_{q}\left(\frac{1}{q}-\log \left(1+\frac{1}{q}\right)\right)
$$


then we obtain that

$$
\begin{aligned}
H+\sum_{q}\left(\frac{1}{q}-\log \left(1+\frac{1}{q}\right)\right) & =H+\sum_{q}\left(\frac{1}{q}-\log \left(\frac{q+1}{q}\right)\right) \\
& =\sum_{q}\left(\log \left(\frac{q}{q-1}\right)-\frac{1}{q}\right)+\sum_{q}\left(\frac{1}{q}-\log \left(\frac{q+1}{q}\right)\right) \\
& =\sum_{q}\left(\log \left(\frac{q}{q-1}\right)-\log \left(\frac{q+1}{q}\right)\right) \\
& =\sum_{q}\left(\log \left(\frac{q}{q-1}\right)+\log \left(\frac{q}{q+1}\right)\right) \\
& =\sum_{q}\left(\log \left(\frac{q^{2}}{(q-1) \times(q+1)}\right)\right) \\
& =\sum_{q}\left(\log \left(\frac{q^{2}}{\left(q^{2}-1\right)}\right)\right) \\
& =\log \left(\frac{\pi^{2}}{6}\right)
\end{aligned}
$$

according to the theorems 2.1 and 2.6. Therefore, the proof is done.

\section{A Simple Case}

We can easily prove that Robins $(n)$ is true for certain kind of numbers:

Lemma 4.1. Robins $(n)$ holds for all natural numbers $n>5040$ when $q \leq 5$, where $q$ is the largest prime divisor of $n$.

Proof. Let $n>5040$ and let all its prime divisors be $q_{1}<\cdots<q_{m} \leq 5$, then we need to prove

$$
f(n)<e^{\gamma} \times \log \log n
$$

that is true when

$$
\prod_{i=1}^{m} \frac{q_{i}}{q_{i}-1} \leq e^{\gamma} \times \log \log n
$$

according to the theorem 2.5. For the prime divisors $q_{1}<\cdots<q_{m} \leq 5$,

$$
\prod_{i=1}^{m} \frac{q_{i}}{q_{i}-1} \leq \frac{2 \times 3 \times 5}{1 \times 2 \times 4}=3.75<e^{\gamma} \times \log \log (5040) \approx 3.81 .
$$

For all natural numbers $n>5040$, we note that

$$
e^{\gamma} \times \log \log (5040)<e^{\gamma} \times \log \log n
$$

and therefore, the proof is complete when $q_{1}<\cdots<q_{m} \leq 5$. 


\section{The Function $\varpi(x)$}

Lemma 5.1. The inequality $\varpi(p)>u(p)$ is satisfied for a prime number $p \geq 3$ if and only if Nicolas $(p)$ holds.

Proof. We start from the inequality:

$$
\varpi(p)>u(p)
$$

which is equivalent to

$$
\left(\sum_{q \leq p} \frac{1}{q}-\log \log \theta(p)-B\right)>\sum_{q>p}\left(\log \left(\frac{q}{q-1}\right)-\frac{1}{q}\right) .
$$

We add the following formula to the both sides of the inequality,

$$
\sum_{q \leq p}\left(\log \left(\frac{q}{q-1}\right)-\frac{1}{q}\right)
$$

and due to the theorem 2.1, we obtain that

$$
\sum_{q \leq p} \log \left(\frac{q}{q-1}\right)-\log \log \theta(p)-B>H
$$

because of

$$
H=\sum_{q \leq p}\left(\log \left(\frac{q}{q-1}\right)-\frac{1}{q}\right)+\sum_{q>p}\left(\log \left(\frac{q}{q-1}\right)-\frac{1}{q}\right)
$$

and

$$
\sum_{q \leq p} \log \left(\frac{q}{q-1}\right)=\sum_{q \leq p} \frac{1}{q}+\sum_{q \leq p}\left(\log \left(\frac{q}{q-1}\right)-\frac{1}{q}\right) .
$$

We distribute it and remove $B$ from the both sides:

$$
\sum_{q \leq p} \log \left(\frac{q}{q-1}\right)>\gamma+\log \log \theta(p)
$$

since $H=\gamma-B$. If we apply the exponentiation to the both sides of the inequality, then we have that

$$
\prod_{q \leq p} \frac{q}{q-1}>e^{\gamma} \times \log \theta(p)
$$

which means that Nicolas $(p)$ holds. The same happens in the reverse implication.

Lemma 5.2. The Riemann hypothesis is true if and only if the inequality $\varpi(p)>u(p)$ is satisfied for all prime numbers $p \geq 3$.

Proof. This is a direct consequence of theorems 1.3 and 5.1.

\section{Lemma 5.3.}

$$
\lim _{x \rightarrow \infty} \varpi(x)=0 .
$$

Proof. We know that $\lim _{x \rightarrow \infty} \varpi(x)=0$ for the limits $\lim _{x \rightarrow \infty} \delta(x)=0$ and $\lim _{x \rightarrow \infty} \frac{\theta(x)}{x}=1$. In this way, this is a consequence from the theorems 2.2 and 2.3. 


\section{Inequalities on Hardy-Ramanujan integers}

Lemma 6.1. Let $\prod_{i=1}^{m} q_{i}^{a_{i}}$ be the representation of an Hardy-Ramanujan integer $n>5040$ as a product of the first $m$ primes $q_{1}<\cdots<q_{m}$ with natural numbers as exponents $a_{1} \geq a_{2} \geq \cdots \geq$ $a_{m} \geq 0$. If Robins $(n)$ does not hold, then Nicolas $\left(q_{m}\right)$ holds indeed.

Proof. When Robins $(n)$ does not hold, then

$$
f(n) \geq e^{\gamma} \times \log \log n
$$

Let's assume that Nicolas $\left(q_{m}\right)$ does not hold as well. Consequently,

$$
\prod_{q \leq q_{m}} \frac{q}{q-1} \leq e^{\gamma} \times \log \log N_{m} .
$$

According to the theorem 2.5,

$$
\begin{aligned}
e^{\gamma} \times \log \log N_{m} & \geq \prod_{q \leq q_{m}} \frac{q}{q-1} \\
& >f(n) \\
& \geq e^{\gamma} \times \log \log n .
\end{aligned}
$$

However, this implies that $N_{m}>n$ which is a contradiction since $n>5040$ is an HardyRamanujan integer.

\section{When the Nicolas inequality may fail}

Lemma 7.1. If some prime number $q_{n}>2$ complies with

$$
X_{n} \leq \frac{e^{\gamma} \times 6}{\pi^{2}}
$$

then Nicolas $\left(q_{n}\right)$ does not hold.

Proof. If we have the inequality

$$
X_{n} \leq \frac{e^{\gamma} \times 6}{\pi^{2}}
$$

then this is equivalent to

$$
\prod_{q \leq q_{n}} \frac{q+1}{q} \leq \frac{e^{\gamma} \times 6}{\pi^{2}} \times \log \theta\left(q_{n}\right) .
$$

If we multiply the both sides by $\frac{\pi^{2}}{6}$, so

$$
\frac{\pi^{2}}{6} \times \prod_{q \leq q_{n}} \frac{q+1}{q} \leq e^{\gamma} \times \log \theta\left(q_{n}\right) .
$$

We use that theorem 2.6 to show that

$$
\frac{\pi^{2}}{6} \times \prod_{q \leq q_{n}} \frac{q+1}{q}>\left(\prod_{\substack{q \leq q_{n} \\ 7}} \frac{q^{2}}{q^{2}-1}\right) \times \prod_{q \leq q_{n}} \frac{q+1}{q} .
$$


Besides,

$$
\left(\prod_{q \leq q_{n}} \frac{q^{2}}{q^{2}-1}\right) \times \prod_{q \leq q_{n}} \frac{q+1}{q}=\prod_{q \leq q_{n}} \frac{q}{q-1}
$$

because of

$$
\frac{q}{q-1}=\frac{q^{2}}{q^{2}-1} \times \frac{q+1}{q}
$$

Consequently, we obtain that

$$
\prod_{q \leq q_{n}} \frac{q}{q-1} \leq e^{\gamma} \times \log \theta\left(q_{n}\right)
$$

and therefore, $\operatorname{Nicolas}\left(q_{n}\right)$ does not hold.

\section{Main Insight}

The next lemma is a main insight.

Lemma 8.1. Let $\frac{\pi^{2}}{6} \times \log \log n^{\prime} \leq \log \log n$ for some natural number $n>5040$ such that $n^{\prime}$ is the square free kernel of the natural number $n$. Then $\operatorname{Robins}(n)$ holds.

Proof. Let $n^{\prime}$ be the square free kernel of the natural number $n$, that is the product of the distinct primes $q_{1}, \ldots, q_{m}$. By assumption we have that

$$
\frac{\pi^{2}}{6} \times \log \log n^{\prime} \leq \log \log n
$$

For all square free $n^{\prime} \leq 5040$, Robins $\left(n^{\prime}\right)$ holds if and only if $n^{\prime} \notin\{2,3,5,6,10,30\}$ [1]. Robins $(n)$ holds for all natural numbers $n>5040$ when $n^{\prime} \in\{2,3,5,6,10,15,30\}$ due to the lemma 4.1. When $n^{\prime}>5040$, we know that Robins $\left(n^{\prime}\right)$ holds and so

$$
f\left(n^{\prime}\right)<e^{\gamma} \times \log \log n^{\prime}
$$

because of the theorem 1.5. By the previous lemma 3.1:

$$
f(n)<\frac{\pi^{2}}{6} \times \prod_{i=1}^{m} \frac{q_{i}+1}{q_{i}} .
$$

Suppose by way of contradiction that $\operatorname{Robins}(n)$ fails. Then

$$
f(n) \geq e^{\gamma} \times \log \log n .
$$

We claim that

$$
\frac{\pi^{2}}{6} \times \prod_{i=1}^{m} \frac{q_{i}+1}{q_{i}}>e^{\gamma} \times \log \log n
$$

Since otherwise we would have a contradiction. This shows that

$$
\frac{\pi^{2}}{6} \times \prod_{i=1}^{m} \frac{q_{i}+1}{q_{i}}>\frac{\pi^{2}}{6} \times e^{\gamma} \times \log \log n^{\prime} .
$$


Thus

$$
\prod_{i=1}^{m} \frac{q_{i}+1}{q_{i}}>e^{\gamma} \times \log \log n^{\prime}
$$

and

$$
\prod_{i=1}^{m} \frac{q_{i}+1}{q_{i}}>f\left(n^{\prime}\right)
$$

This is a contradiction since $f\left(n^{\prime}\right)$ is equal to

$$
\frac{\left(q_{1}+1\right) \times \cdots \times\left(q_{m}+1\right)}{q_{1} \times \cdots \times q_{m}}
$$

according to the formula $f(x)$ for the square free numbers [1].

\section{Pros for the Riemann Hypothesis}

Theorem 9.1. The Riemann hypothesis is possibly true.

Proof. Let $\prod_{i=1}^{m} q_{i}^{a_{i}}$ be the representation of a sufficiently large Hardy-Ramanujan integer $n>$ 5040 as a product of the first $m$ primes $q_{1}<\cdots<q_{m}$ with natural numbers as exponents $a_{1} \geq a_{2} \geq \cdots \geq a_{m} \geq 0$. We claim that for every sufficiently large Hardy-Ramanujan integer $n>5040$, then Robins $(n)$ could always hold. Suppose that Robins $(n)$ does not hold and so, the Riemann hypothesis would be false. Hence,

$$
f(n) \geq e^{\gamma} \times \log \log n .
$$

We use that theorem 2.4,

$$
\left(\prod_{i=1}^{m} \frac{q_{i}}{q_{i}-1}\right) \times \prod_{i=1}^{m}\left(1-\frac{1}{q_{i}^{a_{i}+1}}\right) \geq e^{\gamma} \times \log \log n
$$

which is equivalent to

$$
\left(\prod_{i=1}^{m} \frac{q_{i}^{2}}{q_{i}^{2}-1}\right) \times\left(\prod_{i=1}^{m} \frac{q_{i}+1}{q_{i}}\right) \times \prod_{i=1}^{m}\left(1-\frac{1}{q_{i}^{a_{i}+1}}\right) \geq e^{\gamma} \times \log \log n .
$$

If we divide the both sides by $\log \log N_{m}$, then we obtain

$$
\left(\prod_{i=1}^{m} \frac{q_{i}^{2}}{q_{i}^{2}-1}\right) \times X_{m} \times \prod_{i=1}^{m}\left(1-\frac{1}{q_{i}^{a_{i}+1}}\right) \geq e^{\gamma} \times \frac{\log \log n}{\log \log N_{m}}
$$

because of $\log \log N_{m}=\log \theta\left(q_{m}\right)$, where $N_{m}$ is the primorial number of order $m$. We know that $X_{m} \leq \frac{e^{\gamma} \times 6}{\pi^{2}}$ is false according to the lemmas 6.1 and 7.1. From the lemma 3.2, we know that there exists a natural number $N$ such that $X_{m}<\frac{e^{\gamma} \times 6}{\pi^{2}}+\varepsilon$ for all natural numbers $m>N$ and for a positive real number $\varepsilon<\frac{6}{\pi^{2}}$. Moreover, only a finite number of elements of the sequence are greater than $\frac{e^{\gamma} \times 6}{\pi^{2}}+\varepsilon$ (this could be an empty set). Under our assumption, there exist infinitely many Hardy-Ramanujan integers $n>5040$ such that Robins $(n)$ does not hold and $X_{m}<\frac{e^{\gamma} \times 6}{\pi^{2}}+\varepsilon$. In addition, $q_{m}$ cannot have an upper bound under our assumption. In general, if $q_{m}$ would have 
an upper bound, then our assumption fails as a consequence of the lemma 8.1. In this way, we obtain that

$$
\left(\prod_{i=1}^{m} \frac{q_{i}^{2}}{q_{i}^{2}-1}\right) \times\left(\frac{e^{\gamma} \times 6}{\pi^{2}}+\varepsilon\right) \times \prod_{i=1}^{m}\left(1-\frac{1}{q_{i}^{a_{i}+1}}\right) \geq e^{\gamma} \times \frac{\log \log n}{\log \log N_{m}}
$$

which is the same as

$$
\left(\prod_{i=1}^{m} \frac{q_{i}^{2}}{q_{i}^{2}-1}\right) \times \frac{6}{\pi^{2}} \times\left(e^{\gamma}+c\right) \times \prod_{i=1}^{m}\left(1-\frac{1}{q_{i}^{a_{i}+1}}\right) \geq e^{\gamma} \times \frac{\log \log n}{\log \log N_{m}}
$$

for a sufficiently small positive value of $c=\varepsilon \times \frac{\pi^{2}}{6}$. That would be equivalent to

$$
\left(\prod_{q>q_{m}} \frac{q^{2}-1}{q^{2}}\right) \times\left(e^{\gamma}+c\right) \times \prod_{i=1}^{m}\left(1-\frac{1}{q_{i}^{a_{i}+1}}\right) \geq e^{\gamma} \times \frac{\log \log n}{\log \log N_{m}} .
$$

Since $n$ is an Hardy-Ramanujan integer, then

$$
\left(\prod_{q>q_{m}} \frac{q^{2}-1}{q^{2}}\right) \times \prod_{i=1}^{m}\left(1-\frac{1}{q_{i}^{a_{i}+1}}\right)<\prod_{q}\left(1-\frac{1}{q^{a_{1}+1}}\right)=\frac{1}{\zeta\left(a_{1}+1\right)}
$$

because of the theorem 2.7, where $a_{1}$ is the highest exponent such that $2^{a_{1}} \mid n$. Therefore,

$$
\frac{\left(e^{\gamma}+c\right)}{\zeta\left(a_{1}+1\right)}>e^{\gamma} \times \frac{\log \log n}{\log \log N_{m}}
$$

for a sufficiently small positive value of $0<c<1$. However, this could be false for a sufficiently small positive value of $\varepsilon<\frac{6}{\pi^{2}}$ that we could choose, where $c=\varepsilon \times \frac{\pi^{2}}{6}$ would be a very small positive value as well. In addition, we know that $\frac{\log \log n}{\log \log N_{m}}>1$ due to the theorem 1.5. Furthermore, from the paper [6], we know that Robins $(n)$ holds for all natural numbers $n>5040$ when

$$
a_{1}>\left\lceil\frac{1}{\log 2} \times\left(\left(\log \left(2^{-a_{1}+19} \times n\right)\right)^{\frac{1048576}{1048575}}-\log \left(2^{-a_{1}} \times n\right)\right)\right\rceil .
$$

In conclusion, for every sufficiently large Hardy-Ramanujan integer $n>5040$, then Robins $(n)$ could always hold. By contraposition, the Riemann hypothesis is possibly true, because of the theorems 1.4 and 1.6.

Theorem 9.2. The Riemann hypothesis is possibly true.

Proof. We claim that for every sufficiently large Hardy-Ramanujan integer $n>5040$, then Robins $(n)$ could always hold. Let $\prod_{i=1}^{m} q_{i}^{a_{i}}$ be the representation of a sufficiently large HardyRamanujan integer $n>5040$ as a product of the first $m$ primes $q_{1}<\cdots<q_{m}$ with natural numbers as exponents $a_{1} \geq a_{2} \geq \cdots \geq a_{m} \geq 0$. Suppose that Robins $(n)$ does not hold and so, the Riemann hypothesis would be false. Hence,

$$
f(n) \geq e^{\gamma} \times \log \log n .
$$

We use that theorem 2.4,

$$
\left(\prod_{i=1}^{m} \frac{q_{i}}{q_{i}-1}\right) \times \prod_{i=1}^{m}\left(1-\frac{1}{q_{i}^{a_{i}+1}}\right) \geq e^{\gamma} \times \log \log n
$$


which is equivalent to

$$
\left(\prod_{i=1}^{m} \frac{q_{i}^{2}}{q_{i}^{2}-1}\right) \times\left(\prod_{i=1}^{m} \frac{q_{i}+1}{q_{i}}\right) \times\left(\prod_{i=1}^{m}\left(1-\frac{1}{q_{i}^{a_{i}+1}}\right)\right) \geq e^{\gamma} \times \log \log n .
$$

This is equivalent to

$$
\frac{\log \log N_{m}}{\log \log n} \times\left(\prod_{i=1}^{m} \frac{q_{i}^{2}}{q_{i}^{2}-1}\right) \times\left(\prod_{i=1}^{m} \frac{q_{i}+1}{q_{i}}\right) \times\left(\prod_{i=1}^{m}\left(1-\frac{1}{q_{i}^{a_{i}+1}}\right)\right) \geq e^{\gamma} \times \log \log N_{m}
$$

where $N_{m}$ is the primorial number of order $m$. If we apply the logarithm to the both sides of the inequality, then

$\log \left(\frac{\log \log N_{m}}{\log \log n}\right)+\log \left(\prod_{i=1}^{m} \frac{q_{i}^{2}}{q_{i}^{2}-1}\right)+\log \left(\prod_{i=1}^{m} \frac{q_{i}+1}{q_{i}}\right)+\log \left(\prod_{i=1}^{m}\left(1-\frac{1}{q_{i}^{a_{i}+1}}\right)\right) \geq \gamma+\log \log \theta\left(q_{m}\right)$

because of $\log N_{m}=\theta\left(q_{m}\right)$. Let's multiply by -1 the both sides of the inequality,

$\log \left(\frac{\log \log n}{\log \log N_{m}}\right)-\log \left(\prod_{i=1}^{m} \frac{q_{i}^{2}}{q_{i}^{2}-1}\right)-\log \left(\prod_{i=1}^{m} \frac{q_{i}+1}{q_{i}}\right)+\log \left(\prod_{i=1}^{m}\left(\frac{q_{i}^{a_{i}+1}}{q_{i}^{a_{i}+1}-1}\right)\right) \leq-\gamma-\log \log \theta\left(q_{m}\right)$

which is equivalent to

$$
\begin{gathered}
\log \left(\frac{\log \log n}{\log \log N_{m}}\right)-\log \left(\prod_{i=1}^{m} \frac{q_{i}^{2}}{q_{i}^{2}-1}\right)+\left(\sum_{q \leq q_{m}} \frac{1}{q}\right)-\log \left(\prod_{i=1}^{m} \frac{q_{i}+1}{q_{i}}\right)+\log \left(\prod_{i=1}^{m}\left(\frac{q_{i}^{a_{i}+1}}{q_{i}^{a_{i}+1}-1}\right)\right) \\
\leq\left(\sum_{q \leq q_{m}} \frac{1}{q}\right)-\gamma-\log \log \theta\left(q_{m}\right)
\end{gathered}
$$

after adding $\sum_{q \leq q_{m}} \frac{1}{q}$ to the both sides of the inequality. This the same as

$$
\begin{gathered}
\log \left(\frac{\log \log n}{\log \log N_{m}}\right)-\log \left(\prod_{i=1}^{m} \frac{q_{i}^{2}}{q_{i}^{2}-1}\right)+\sum_{q}\left(\frac{1}{q}-\log \left(1+\frac{1}{q}\right)\right)-\sum_{q>q_{m}}\left(\frac{1}{q}-\log \left(1+\frac{1}{q}\right)\right) \\
+\log \left(\prod_{i=1}^{m}\left(\frac{q_{i}^{a_{i}+1}}{q_{i}^{a_{i}+1}-1}\right)\right) \leq \varpi\left(q_{m}\right)-H
\end{gathered}
$$

which is

$$
\begin{gathered}
\log \left(\frac{\log \log n}{\log \log N_{m}}\right)-\log \left(\prod_{i=1}^{m} \frac{q_{i}^{2}}{q_{i}^{2}-1}\right)-\sum_{q>q_{m}}\left(\frac{1}{q}-\log \left(1+\frac{1}{q}\right)\right)+\log \left(\frac{\pi^{2}}{6}\right)+\log \left(\prod_{i=1}^{m}\left(\frac{q_{i}^{a_{i}+1}}{q_{i}^{a_{i}+1}-1}\right)\right) \\
\leq u\left(q_{m}\right)+\varepsilon
\end{gathered}
$$

due to the definition 1.7 and the theorem 2.1 with the lemmas 5.1, 5.3, 6.1 and 3.3, where $\varepsilon=$ $\varpi\left(q_{m}\right)-u\left(q_{m}\right)$ could be a sufficiently small positive real number that goes to 0 when $q_{m}$ tends to infinity. Certainly, we would have that

$$
\lim _{m \rightarrow \infty} \varpi\left(q_{m}\right)-u\left(q_{m}\right)=0
$$


because of

$$
\lim _{m \rightarrow \infty} \varpi\left(q_{m}\right)=0
$$

and

$$
\lim _{m \rightarrow \infty} u\left(q_{m}\right)=\lim _{m \rightarrow \infty} \frac{1}{2 \times\left(q_{m}-1\right)}=0
$$

since $0<u\left(q_{m}\right) \leq \frac{1}{2 \times\left(q_{m}-1\right)}$ according to the theorem 2.8 and the lemma 5.3. Actually, $q_{m}$ cannot have an upper bound under our assumption, so the positive value $\varepsilon$ gets smaller and smaller as the chosen Hardy-Ramanujan integer $n$ grows. In general, if $q_{m}$ would have an upper bound, then our assumption fails as a consequence of the lemma 8.1: our assumption is that there would be infinitely many natural numbers $n>5040$ which are an Hardy-Ramanujan integer and counterexample of the Robin inequality. We know that

$$
\begin{aligned}
& u\left(q_{m}\right)+\sum_{q>q_{m}}\left(\frac{1}{q}-\log \left(1+\frac{1}{q}\right)\right)+\log \left(\prod_{i=1}^{m} \frac{q_{i}^{2}}{q_{i}^{2}-1}\right) \\
& =\sum_{q>q_{m}}\left(\log \left(\frac{q}{q-1}\right)-\frac{1}{q}\right)+\sum_{q>q_{m}}\left(\frac{1}{q}-\log \left(1+\frac{1}{q}\right)\right)+\log \left(\prod_{i=1}^{m} \frac{q_{i}^{2}}{q_{i}^{2}-1}\right) \\
& =\sum_{q>q_{m}}\left(\log \left(\frac{q}{q-1}\right)-\log \left(1+\frac{1}{q}\right)\right)+\log \left(\prod_{i=1}^{m} \frac{q_{i}^{2}}{q_{i}^{2}-1}\right) \\
& =\sum_{q>q_{m}}\left(\log \left(\frac{q}{q-1}\right)+\log \left(\frac{q}{q+1}\right)\right)+\log \left(\prod_{i=1}^{m} \frac{q_{i}^{2}}{q_{i}^{2}-1}\right) \\
& =\sum_{q>q_{m}}\left(\log \left(\frac{q^{2}}{q^{2}-1}\right)\right)+\log \left(\prod_{i=1}^{m} \frac{q_{i}^{2}}{q_{i}^{2}-1}\right) \\
& =\sum_{q}\left(\log \left(\frac{q^{2}}{q^{2}-1}\right)\right) \\
& =\log \left(\frac{\pi^{2}}{6}\right)
\end{aligned}
$$

using the theorem 2.6. It is enough to distribute and remove the value of $\log \left(\frac{\pi^{2}}{6}\right)$ from the both sides to show that

$$
\log \left(\frac{\log \log n}{\log \log N_{m}}\right)+\log \left(\prod_{i=1}^{m}\left(\frac{q_{i}^{a_{i}+1}}{q_{i}^{a_{i}+1}-1}\right)\right) \leq \varepsilon
$$

which is equivalent to

$$
\left(\frac{\log \log n}{\log \log N_{m}}\right) \times \prod_{i=1}^{m}\left(\frac{q_{i}^{a_{i}+1}}{q_{i}^{a_{i}+1}-1}\right) \leq e^{\varepsilon} .
$$

However, this could be false for a sufficiently small positive value of $\varepsilon$, since we know that $\varepsilon$ tends to 0 as $n$ grows. In addition, we know that $\frac{\log \log n}{\log \log N_{m}}>1$ due to the theorem 1.5. In conclusion, for every sufficiently large Hardy-Ramanujan integer $n>5040$, then Robins $(n)$ could always hold. By contraposition, the Riemann hypothesis is possibly true, because of the theorems 1.4 and 1.6 . 


\section{Acknowledgments}

The author would like to thank Richard J. Lipton and Craig Helfgott for helpful comments and my mother, maternal brother and my friend Sonia for their support.

\section{References}

[1] Y. Choie, N. Lichiardopol, P. Moree, P. Solé, On Robin's criterion for the Riemann hypothesis, Journal de Théorie des Nombres de Bordeaux 19 (2) (2007) 357-372. doi:10.5802/jtnb.591.

[2] J.-L. Nicolas, Petites valeurs de la fonction d'Euler, Journal of number theory 17 (3) (1983) $375-388$ doi:10.1016/0022-314X(83)90055-0

[3] G. Robin, Grandes valeurs de la fonction somme des diviseurs et hypothèse de Riemann, J. Math. pures appl 63 (2) (1984) 187-213.

[4] F. Mertens, Ein Beitrag zur analytischen Zahlentheorie., J. reine angew. Math. 1874 (78) (1874) 46-62. doi:10.1515/crll.1874.78.46.

URL https://doi.org/10.1515/crll.1874.78.46

[5] T. H. Grönwall, Some asymptotic expressions in the theory of numbers, Transactions of the American Mathematical Society 14 (1) (1913) 113-122. doi:10.2307/1988773.

[6] A. Hertlein, Robin's inequality for new families of integers, at https://arxiv.org/pdf/1612.05186v1.pdf (December 2016). arXiv: 1612.05186.

[7] H. M. Edwards, Riemann's Zeta Function, Dover Publications, 2001. 